\title{
Values and Risk Perceptions: A Cross-Cultural Examination
}

\author{
Gülbanu Kaptan, ${ }^{1, \dagger, *}$ Shoshana Shiloh, ${ }^{2}$ and Dilek Önkal ${ }^{3}$
}

\begin{abstract}
This article examines the relationship between values and risk perceptions regarding terror attacks. The participants in the study are university students from Turkey $(n=536)$ and Israel $(n=298)$. Schwartz value theory $(1992,1994)$ is applied to conceptualize and measure values. Cognitive (perceived likelihood and perceived severity) and emotional (fear, helplessness, anger, distress, insecurity, hopelessness, sadness, and anxiety) responses about the potential of (i) being personally exposed to a terror attack, and (ii) a terror attack that may occur in one's country are assessed to measure risk perceptions. Comparison of the two groups suggests that the Turkish participants are significantly more emotional about terror risks than the Israeli respondents. Both groups perceive the risk of a terror attack that may occur in their country more likely than the risk of being personally exposed to a terror attack. No significant differences are found in emotional representations and perceived severity ratings regarding these risks. Results provide support for the existence of a link between values and risk perceptions of terror attacks. In both countries, self-direction values are negatively related to emotional representations, whereas security values are positively correlated with emotions; hedonism and stimulation values are negatively related to perceived likelihood. Current findings are discussed in relation to previous results, theoretical approaches (the social amplification of risk framework and cultural theory of risk), and practical implications (increasing community support for a course of action, training programs for risk communicators).
\end{abstract}

KEY WORDS: Cross-cultural comparisons; risk perception; terror; values

\section{INTRODUCTION}

The 9/11 attacks in the United States and subsequent terrorist bombings in a number of European cities including Istanbul, Madrid, and London have been instrumental in raising people's awareness about terrorism. The threat of a terror attack can be very affectively arousing. ${ }^{(1)}$ The social amplification

\footnotetext{
${ }^{1}$ Centre for Rural Economy, Newcastle University, Newcastle upon Tyne, UK.

${ }^{2}$ Department of Psychology, Tel Aviv University, Tel Aviv, Israel.

${ }^{3}$ Faculty of Business Administration, Bilkent University, Ankara, Turkey.

${ }^{\dagger}$ At the time of this research was a PhD student at the Faculty of Business Administration, Bilkent University, Ankara, Turkey.

*Address correspondence to Gülbanu Kaptan, Newcastle University, Centre for Rural Economy, Newcastle upon Tyne, NE1 7RU, UK; gulbanu.kaptan@newcastle.ac.uk.
}

of risk framework (SARF) states that certain kinds of hazards and accidents characterized by deaths, injuries, damage, and social disruption are especially likely to cause widespread and strong concerns. ${ }^{(2)}$ These concerns may in turn lead to severe consequences. For example, it has been argued that after the attacks of 9/11 in the United States, death toll by car accidents has increased, mainly due to switching of preferences from flying to driving in avoidance of terror risk. ${ }^{(3)}$ A crucial role of social sciences in risk management is to translate research findings into applicable and useful suggestions regarding communication of risk information. ${ }^{(4)}$ Therefore, it is important for risk communicators to understand the complexity of factors influencing risk perceptions, so that their messages could be particularly tailored to specific individuals or target groups. These factors 
include people's beliefs, attitudes, judgments, and feelings, in addition to their social/cultural values and their dispositions toward hazards and related benefits. $^{(5)}$ Identifying and understanding their variability within and between groups may have important implications, such as increasing community support for proposed course of actions.

Cultural theory of risk $(\mathrm{CTR})^{(6)}$ and $\mathrm{SARF}^{(2)}$ have made important contributions to the risk perception literature by emphasizing the role of social/cultural processes. The CTR conceptualizes risk as a collective phenomenon in which every cultural group chooses to attend to some risks and ignore others to maintain their particular way of life. Building on the earlier CTR studies, worldviews were measured using attitudinal survey techniques and these findings were correlated to risk perception. ${ }^{(7,8)}$ Shared findings with comparable studies demonstrated a relationship between people's worldviews (i.e., fatalism, hierarchy, individualism, and egalitarianism) and risk perceptions of a wide range of hazards (e.g., nuclear power, genetic engineering, and ozone depletion). ${ }^{\left({ }^{9}-11\right)}$ For example, the egalitarian view was found to have a negative correlation with the support of nuclear power, indicating an antinuclear tendency. ${ }^{(9)}$ SARF, on the other hand, conceives risk as a socially constructed phenomenon shaped by individuals and groups across different cultural/societal contexts. Social amplification is triggered by the occurrence of an adverse event, such as a terrorist bombing. The detrimental impact of such an event sometimes extends far beyond the direct damages to victims and property through the process of risk amplification.

Based on the theoretical background linking risk to social factors, this study focuses on exploring the relationships between basic values and risk perceptions of terror attacks in two countries, Turkey and Israel. These two countries share an ongoing threat of terror attacks, but differ in their sociocultural backgrounds. ${ }^{(12)}$ To conceptualize and measure values, we use Schwartz value theory (SVT), ${ }^{(13,14)}$ which defines 10 types of values: power, achievement, hedonism, stimulation, self-direction, universalism, benevolence, tradition, conformity, and security. Each of them represents a distinct motivational goal that guides individuals to satisfy one or more of the three universal requirements of all individuals and societies: needs of individuals as biological organisms, requisites of coordinated social interaction, and requirements for the smooth functioning and survival of groups. For example, self-direction represents

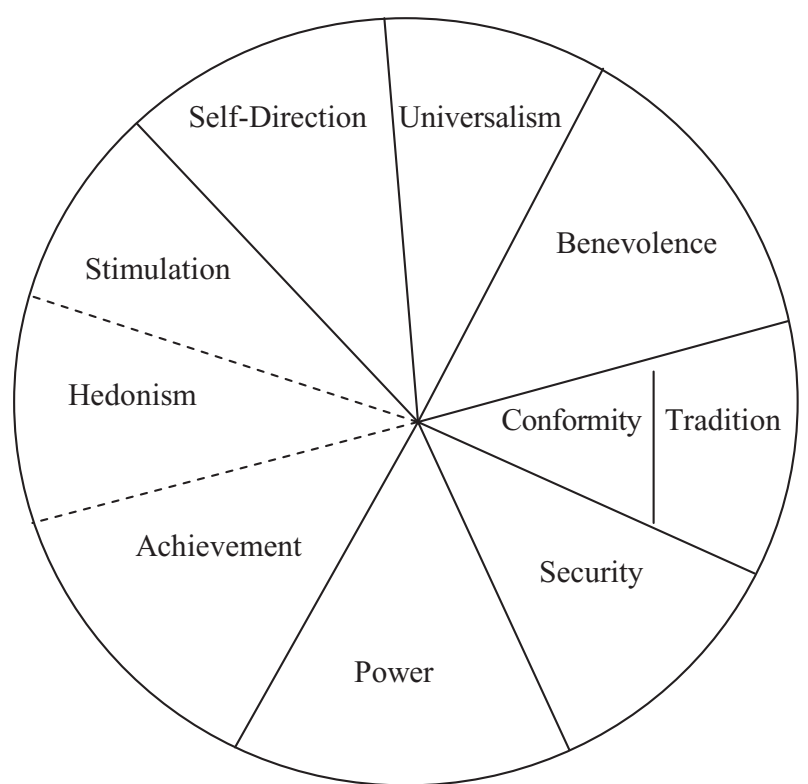

Fig. 1. Theoretical model of relations among motivational types of values (with permission, from Schwartz, 1992).

the motivational goal of independent thought and action, choosing, creating, and exploring. It derives from organismic needs for control and mastery and interactional requirements of autonomy and independence. Creativity, freedom, choosing own goals, curious, independent, self-respect, intelligent, and privacy values make the meaning of self-direction more concrete and explicit. The definitions of the value types are presented in Table I.

The SVT specifies the structure of congruent and competing relations among values, as presented in Fig. 1. Congruent values are closer in either direction around the circle because they express similar motivational goals, whereas competing values are distant because they represent antagonistic goals. For example, self-direction and stimulation are adjacent as they both represent intrinsic interest in novelty and mastery (congruent values). However, self-direction and tradition are distant because of the contrast between novelty and preservation of time-honored customs (competing values). The circular structure stimulates hypothesizing about the relations of values to other variables in an integrated manner. The SVT was empirically assessed among 210 samples from 67 countries located on every inhabited continent. ${ }^{(15)}$ In the vast majority of samples, both the distinctiveness of the 10 values and the structure of their relations have been verified. ${ }^{(16)}$ 
Table I. Definition of Value Types (with Permission, from Schwartz, 1994)

Power: Social status and prestige, control or dominance over people and resources (authority, social power, wealth, preserving one's public image)

Achievement: Personal success through demonstrating competence according to social standards (ambitious, successful, capable, influential)

Hedonism: Pleasure or sensuous gratification for oneself (pleasure, enjoying life, self-indulgent)

Stimulation: excitement, novelty, and challenge in life (daring a varied life, an exciting life)

Self-Direction: Independent thought and action, choosing, creating, exploring (creativity, freedom, independent, choosing own goals, curious)

Universalism: Understanding, appreciation, tolerance, and protection for the welfare of all people and for nature (equality, social justice, wisdom, broad-minded, protecting the environment, unity with nature, a world of beauty)

Benevolence: Preservation and enhancement of the welfare of people with whom one is in frequent personal contact (helpful, honest, forgiving, loyal, responsible)

Tradition: Respect, commitment, and acceptance of the customs and ideas that traditional culture or religion provide (devout, respect for tradition, humble, moderate)

Conformity: Restraint of actions, inclinations, and impulses likely to upset or harm others and violate social expectations and norms (self-discipline, politeness, honoring parents and elders, obedience)

Security: Safety, harmony, and stability of society, of relationships and of self (family security, national security, social order, clean, reciprocation of favors)

In one study, relations of people's values to their microworries (about self and its extensions) and macroworries (about society and the world) were investigated. ${ }^{(17)}$ The findings showed that values explained approximately twice as much variance in macroworries as in microworries regarding direct and salient threats. It was suggested that these differences result from the fact that individual differences in coping ability are more likely than values to influence the incidence and intensity of microworries. Terror risk can be perceived both at micro and macro levels. If the person is being exposed to a terror attack, it may be perceived at the individual (micro) level. If the risk is a terror attack that may occur in one's country, it may be perceived at the societal (macro) level. In a similar vein, it has been claimed that several individual and wider group-level factors are likely to underlie how individuals perceive terror threats. ${ }^{(18)}$ Therefore, the current research examines the relationships between values and risk perceptions both at individual and societal levels.

The risk of a terror attack may be viewed as a threat to the fulfillment of all three universal requirements of individuals and societies (i.e., biological survival, coordinated social interaction, and functioning and survival of the group) noted in the SVT. For example, perceived high risk of a terror attack may prevent an individual from enjoying life, which is a threat to his/her own needs. Productivity at work may be negatively affected, which is a threat to the functioning of the society, and disruptions to social interactions can be caused by defensive and avoidant social behaviors of frightened individuals.
Consequently, people's risk perceptions about terror attacks may be related to the strength of their basic values and may therefore have consequences.

In a previous cross-cultural study between Turkey and Israel, terror risk perception in each sample was described by four independent cognitive factors (i.e., costs, vulnerability, trust, and control) and a single negative emotionality component. ${ }^{(12)}$ This finding supports the view that representations of risk consist of both affective and cognitive variables. ${ }^{(19)}$ In line with this, our study includes one emotional component consisting of eight negative emotions (fear, helplessness, anger, distress, insecurity, hopelessness, sadness, and anxiety) and two cognitive factors (perceived likelihood and perceived severity) that represent terror risk perception. It is in our interest to explore different associations of basic values with emotional representations, perceived likelihood and perceived severity about being personally exposed to a terror attack, and a terror attack that may occur in one's country. Therefore, our research hypotheses about the relationships between values and risk perceptions are postulated in three subsections.

(i) The relationship between values and emotional representations. In a cross-national study examining the relationship between values and worries, self-direction values correlated negatively with microworries (e.g., worries about one's health, safety, and social acceptance). ${ }^{(17)}$ It was also suggested that self-direction values are relatively free of personal anxiety because those who attribute greater importance to self-direction are less likely to attend to uncertainty regarding personal outcomes. ${ }^{(20)}$ 
Accordingly, we predict a relationship between self-direction values and negative emotions. We postulate that the more priority people attribute to self-direction values, the less emotional they might feel about the risk of being personally exposed to a terror attack.

H1: Self-direction values correlate negatively with emotional representations about being personally exposed to a terror attack.

(ii) The relationship between values and perceived likelihood. Self-direction and stimulation values emphasize individuals' own independent thought and action in addition to a tendency to favor change. ${ }^{14)}$ The self with low attachment anxiety, which implicates people's working model of self, how worthy, competent, and socially desirable they perceive themselves to be may lead individuals to engage in autonomous exploration and risk-taking activities. ${ }^{(21)}$ Similarly, hedonism, stimulation, and self-direction values express self-expansion and growth. ${ }^{(20)}$ On the other hand, tradition, conformity, and security values represent deficiency needs such as feeling unsafe, and lacking in control over life in general. ${ }^{(22)}$ Thus, people giving priority to these values may feel more vulnerable to being exposed to a terror attack. Tradition, conformity, and security values also emphasize devotion to one's in-group, in contrast to stimulation values that are characterized by lack of interest in others. ${ }^{(16)} \mathrm{We}$ therefore suggest that people giving priority to tradition, conformity, and security values may perceive the terror risk for their country more likely. However, those attributing more importance to stimulation values may perceive this risk as being less likely:

$\mathrm{H} 2$ : Stimulation and self-direction values correlate negatively with perceived likelihood of being personally exposed to a terror attack, whereas tradition, conformity, and security values correlate positively with perceived likelihood of this risk.

H3: Stimulation values correlate negatively with perceived likelihood of a terror attack that may occur in one's country, whereas tradition, conformity, and security values correlate positively with perceived likelihood of this risk.

(iii) The relationship between values and perceived severity. The more important power values are, the more attention is paid to threats to selfinterests. ${ }^{(17)}$ It may, therefore, be postulated that power values increase severity perceptions about being personally exposed to a terror attack because of its potential disruption of the attainment of personal goals. Conversely, universalism and benevolence values emphasize enhancement of others and transcendence of selfish interests. ${ }^{(15)}$ Hence, people giving priority to these values may perceive consequences of terror attacks as less severe for themselves but more severe for their country.

Security values express the importance people place on personal security, national security, certainty, and structure to avert physical and psychological danger, ambiguity, and risk. ${ }^{(23)}$ Perceived severity about the consequences of a terror attack relates to loss of control over personal and national outcomes that assure security and certainty. Therefore, we postulate a positive relationship between security and perceived severity:

H4: Power and security values correlate positively with perceived severity of being personally exposed to a terror attack, whereas universalism and benevolence values correlate negatively with perceived severity of this risk.

H5: Universalism, benevolence, and security values correlate positively with perceived severity of a terror attack that may occur in one's country.

\section{MATERIAL AND METHODS}

\subsection{Participants}

A total of 650 students at Bilkent and Hacettepe universities (Turkey) and 317 students at Tel Aviv University (Israel) volunteered to participate in the study. The surveys were conducted at Bilkent and Hacettepe universities between October and January 2006, and at Tel Aviv University between March and June 2007. A total of 114 Turkish students and 19 Israeli students, who either rated more than 21 values as having supreme importance as guiding principles in their lives, or any other response more than 35 times, or responded to fewer than 41 values, were excluded from the analysis in compliance with the elimination criteria. ${ }^{(15)}$ As a result, the final sample consisted of 536 Turkish and 298 Israeli students. The Turkish sample included 195 male and 341 female respondents, aged $17-35$ years $(\mathrm{M}=$ 20.74, $S D=2.90$ ); the Israeli sample consisted of 105 male and 193 female respondents, aged 17-40 years $(\mathrm{M}=24.73, S D=3.48)$. 


\subsection{Instruments}

\subsubsection{Values}

The 57-item Schwartz Value Survey (SVS) ${ }^{(13)}$ was administered to measure the importance ratings of specific values representative of 10 universal value types (i.e., power, achievement, hedonism, stimulation, self-direction, universalism, benevolence, conformity, tradition, and security). Participants rated the importance of each item (e.g., equality, respect for tradition, success, and curiosity) "as a guiding principle in my life" on a nine-point scale (ranging from $-1=$ opposed to my principles to $0=$ not important, to $3=$ important, to $7=$ of supreme importance). An importance score for each value was computed by averaging the items that index it. It should be noted that only 46 (of 57) value items with similar meanings across cultures ${ }^{(15)}$ were included in our study. The pattern of relationships among the 46 value items was assessed to examine its correspondence with the theoretical structure presented in Fig. 1. Similarity structure analysis (SSA) ${ }^{(24,25)}$ was applied to map items as points in a multidimensional space, such that the distances between the points reflected the relative interrelations among the items. The SSA maps of the Turkish and Israeli samples were also divided in accordance with the partitioning criteria of Schwartz. ${ }^{(15)}$ Eight basic values for the Turkish sample and seven basic values for the Israeli sample were obtained. Their SSA maps are presented in Figs. 2 and 3, respectively. In both samples, stimulation values formed a joint region with hedonism values, and benevolence values mixed together with conformity values. The organization of specific value items and basic values in both samples was found similar, but not identical, to the theoretical prototype. This finding demonstrates a comprehensive and near universal set of values identified by the SVT. Therefore, any hypothesized relationship between values and risk perception can be examined.

"Stimulation \& hedonism" and "benevolence \& conformity" scores were computed by averaging the items of the combined scales. In the Israeli sample, power and achievement values also mixed together, yielding a "Power \& Achievement" score. The mean scores and reliabilities of the value scales of both samples are presented in Table II. As indicated, the reliabilities (Cronbach $\alpha$ ) of all values except self-direction and security ranged from 0.70 to 0.80 , whereas the reliabilities of these two values ranged from 0.58 to 0.63 .
Table II. Mean Ratings, Standard Deviations, and Reliabilities of Values

\begin{tabular}{|c|c|c|c|}
\hline & $\begin{array}{l}\text { Mean } \\
\text { Rating }\end{array}$ & $\begin{array}{c}\text { Standard } \\
\text { Deviation }\end{array}$ & Reliability \\
\hline \multicolumn{4}{|l|}{ A. Turkish sample $(n=536)$} \\
\hline Power & 3.65 & 1.60 & 0.76 \\
\hline Achievement & 4.47 & 1.19 & 0.71 \\
\hline Hedonism \& Stimulation & 4.14 & 1.13 & 0.73 \\
\hline Self-direction & 5.27 & 0.85 & 0.61 \\
\hline Universalism & 4.74 & 1.00 & 0.80 \\
\hline Benevolence \& Conformity & 4.98 & 0.87 & 0.76 \\
\hline Tradition & 3.33 & 1.25 & 0.72 \\
\hline Security & 5.01 & 1.00 & 0.63 \\
\hline \multicolumn{4}{|l|}{ B. Israeli sample $(n=298)$} \\
\hline Power \& Achievement & 3.71 & 1.00 & 0.74 \\
\hline Hedonism \& Stimulation & 3.95 & 1.01 & 0.77 \\
\hline Self-direction & 5.06 & 0.87 & 0.62 \\
\hline Universalism & 4.32 & 0.88 & 0.70 \\
\hline Benevolence \& Conformity & 4.57 & 0.90 & 0.79 \\
\hline Tradition & 2.91 & 1.16 & 0.68 \\
\hline Security & 4.43 & 1.04 & 0.58 \\
\hline
\end{tabular}

Multivariate analysis of variance was applied to compare the value scores of the Turkish and Israeli samples . Power and achievement scores of the Turkish participants were combined for this analysis $(\mathrm{M}=3.71, S D=1.00, \alpha=0.81)$. The only significant difference was obtained for security values: Turkish participants attributed more importance to security values $(\mathrm{M}=5.01, S D=1.00)$ than their Israeli counterparts $(\mathrm{M}=4.43, S D=1.04, F=15.96, p<0.001)$.

\subsubsection{Risk Perceptions of Terror Attacks}

The Terror Attack Risk Perception Survey (TRPS) was administered to measure the emotional representations and cognitive perceptions of terror attacks. It began with an introductory paragraph that provided brief information on terror attacks in Turkey (in the Turkish version) or in Israel (in the Hebrew version).

Emotional representations (fear, helplessness, anger, distress, insecurity, hopelessness, sadness, and anxiety) were measured by asking respondents about the intensity of their feelings about (1) being personally exposed to a terror attack within a year, and (2) a terror attack that may occur in their countries within a year. These items were taken from the Terror Risk Perception Questionnaire (TRPQ) that had been developed in a previous study comparing Turkey and Israel.(12) Participants reported the intensity of their feelings on seven-point scales 


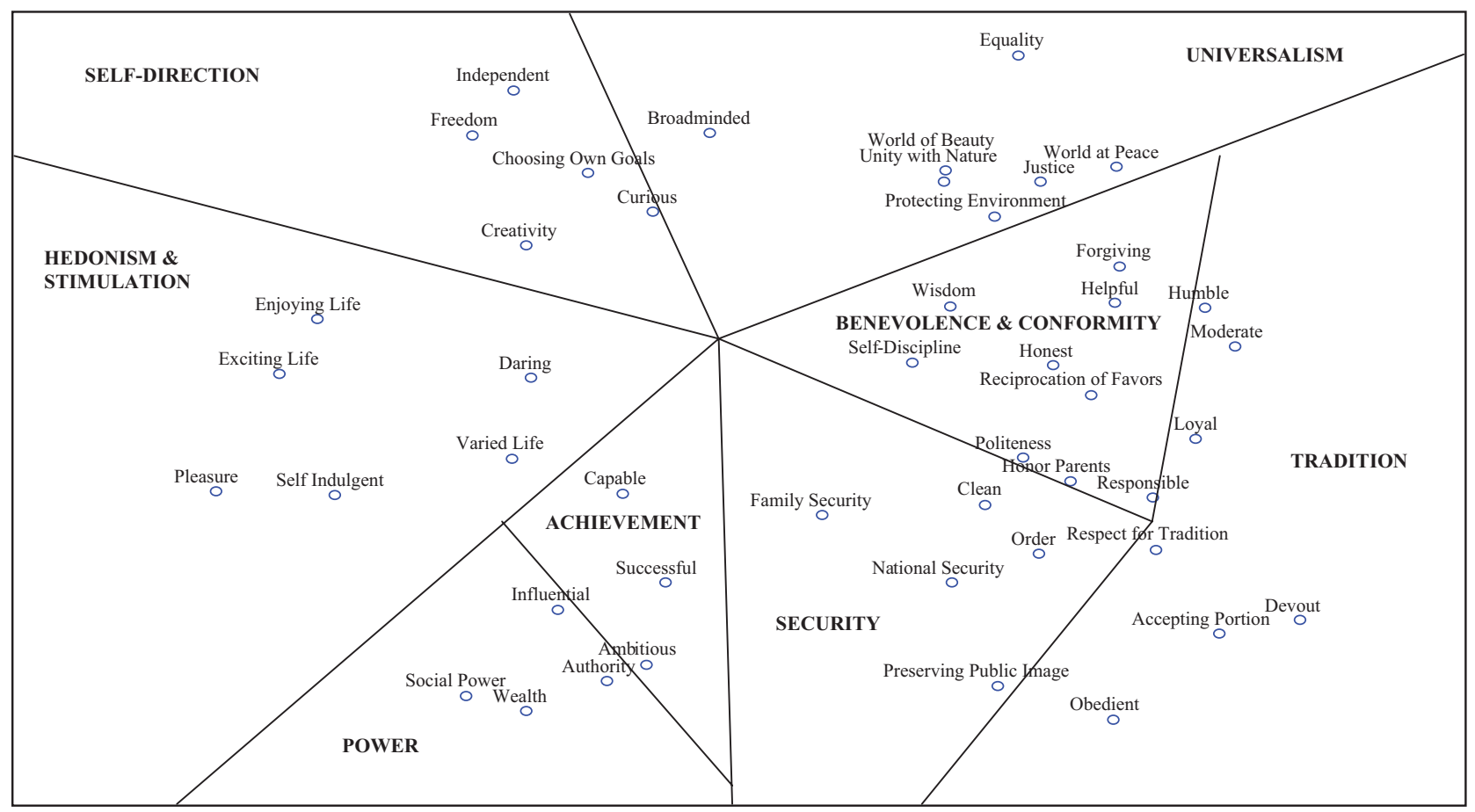

Fig. 2. SSA map of the observed relations among values in the Turkish sample $(n=536$, coefficient of alienation $=0.19)$.

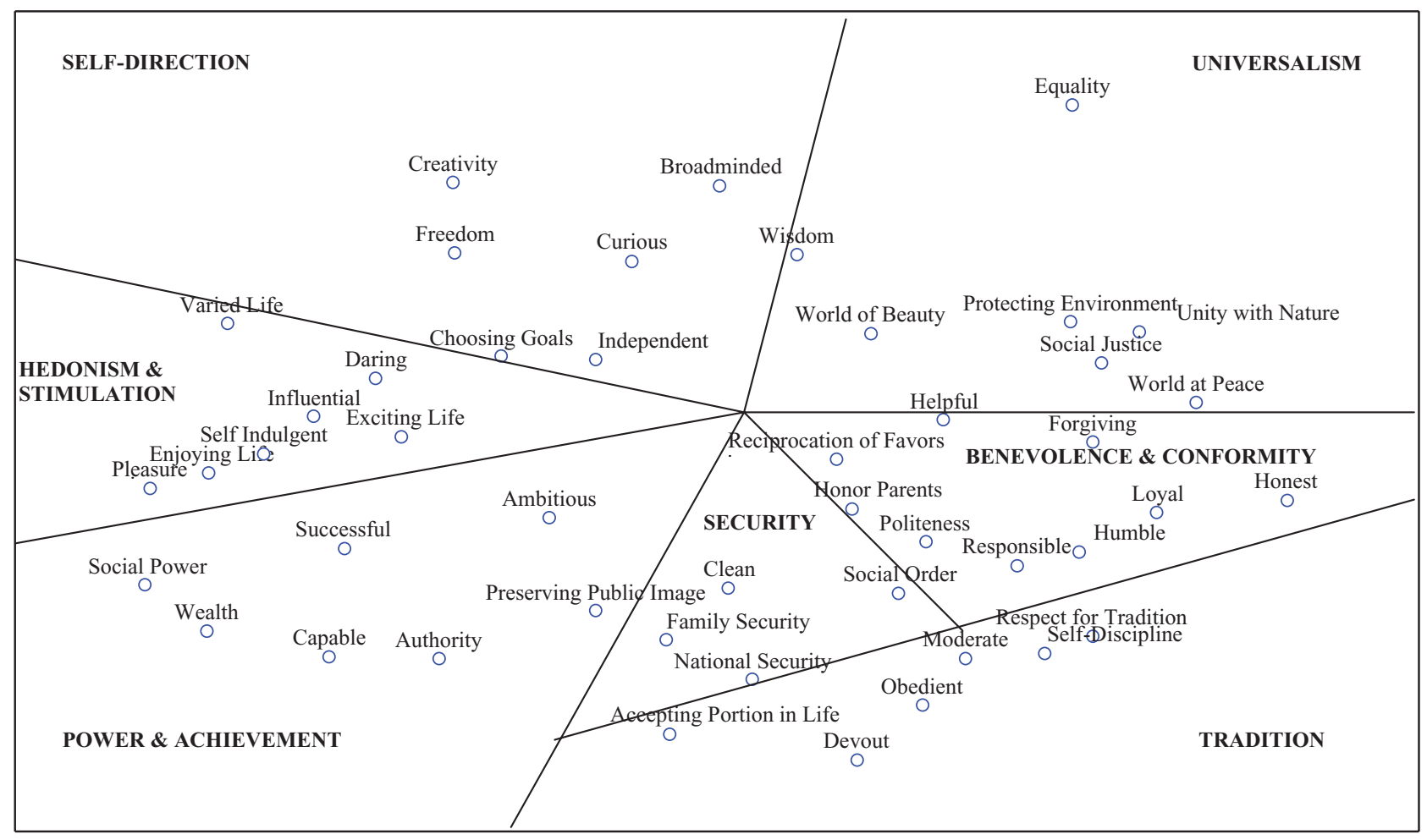

Fig. 3. SSA map of observed relations among values in the Israeli sample $(n=298$, coefficient of alienation $=0.28)$. 
( $1=$ "I do not feel at all," and $7=$ "I strongly feel"). The reliabilities (Chronbach's $\alpha$ ) of the personal and country scales in the Turkish sample were 0.90 and 0.89 , respectively. The reliabilities in the Israeli sample were 0.92 and 0.92 , respectively. The mean ratings across emotional scales were used as emotional representations' scores.

Cognitive perceptions were measured by asking respondents about their perceived likelihood and perceived severity regarding terror attacks. The participants reported their perceived likelihood by using two scales: (1) a seven-point likelihood scale $(1=$ extremely unlikely, and $7=$ extremely likely $)$, asking respondents to indicate how likely they thought they might be personally exposed to a terror attack within a year; and (2) a probability assessment using a percentage scale (with $0 \%=$ "impossible to happen," whereas $100 \%=$ "certain to happen"). The same two scales were repeated for measuring respondents' perceived likelihood/probability about a terror attack that may occur in their country within a year. Pearson correlations between the likelihood and probability scales in the Turkish sample were $r=$ 0.78 and $r=0.43$ for self and country, respectively. In the Israeli sample, the equivalent correlations were $r=0.74$ and $r=0.77$. Perceived likelihood and probability scales were combined into a single likelihood score by averaging them after transformation into standardized scores.

Perceived severity was measured by asking about the severity of consequences resulting from possibly being exposed to a terror attack within a year. Respondents indicated their perceptions on a seven-point scale (ranging from $1=$ "not serious at all" to $7=$ "extremely serious"). They followed a similar scale for judging the severity of a terror attack that may occur in their country within a year.

\subsubsection{Demographic and Background Data}

Variables thought to have potential impact on terror risk perceptions were measured after the introductory paragraph. These included: age, gender, university major, direct personal experience with a terror attack (or through relatives), and perceived knowledge about terror attacks in their own country and in other countries $(1=$ no information at all, $2=$ some information, and $3=a$ lot of information). Significant differences were found between the two cultural groups with respect to age $(t=16.82$, $p<0.001)$ and experience with terror attacks $\left(\chi^{2}=\right.$ $26.08, p<0.001)$. Israeli participants were signif- icantly older and reported more experience with terror attacks than their Turkish counterparts. These differences were taken into account in data analyses.

\subsection{Procedure}

Participants were recruited in response to announcements inviting students to participate in a 20-minute survey about values and risk perception. The surveys were conducted on a volunteer basis. Oral debriefing explaining the purpose of the study along with the names and contact information of the researchers was given to the Turkish participants before filling out the surveys. A similar debriefing was given to the Israeli participants in a written format. The risk perception survey was initially constructed in Turkish and translated into English and Hebrew. Translations were checked by backward procedures using people fluent in the relevant languages. The participants filled out the SVS and risk perception survey in their native language. Filling out the questionnaires took approximately 20 minutes.

\section{RESULTS}

We first present the findings regarding the associations of demographic and background variables with terror risk perceptions in both samples. We then provide and compare risk perceptions in the two samples. Finally, we introduce the results about the relationships between values and risk perceptions in both groups.

\subsection{Associations of Demographic and Background Variables with Risk Perceptions}

Age, experience with terror attacks, and perceived knowledge of terror attacks (in other countries) were unrelated to risk perceptions in the Turkish sample. However, Turkish participants who reported having greater knowledge about terror attacks in Turkey perceived a terror attack that may occur in their country as being more likely $(\mathrm{M}=5.80, S D=1.72)$ than those with less perceived knowledge $(\mathrm{M}=5.03, S D=1.58, F=18.26$, $p<0.001)$. In the Israeli sample, age and perceived knowledge of terror attacks (in other countries) were unrelated to risk perceptions. However, participants who reported having greater knowledge about terror attacks in Israel perceived the severity of a terror attack that may occur in their country as being lower $(\mathrm{M}=4.56, S D=1.56)$ than those with less 
Table III. Gender Differences in Mean Ratings of TRPS Scores

\begin{tabular}{|c|c|c|c|c|c|c|}
\hline & \multicolumn{3}{|c|}{ Being Exposed to a Terror Attack } & \multicolumn{3}{|c|}{ A Terror Attack in Turkey } \\
\hline & Male $(n=195)$ & Female $(n=341)$ & $F$-Statistic & Male $(n=195)$ & Female $(n=341)$ & $F$-Statistic \\
\hline \multicolumn{7}{|l|}{ A. Turkish sample $(n=536)$} \\
\hline Emotional representations & 3.43 & 4.32 & $53.68^{* *}$ & 4.12 & 4.97 & $57.53^{* *}$ \\
\hline Likelihood & 2.79 & 3.35 & $22.06^{* *}$ & 5.74 & 5.40 & 0.89 \\
\hline \multirow[t]{3}{*}{ Severity } & 5.79 & 6.28 & $22.26^{* *}$ & 5.52 & 6.34 & $52.81^{* *}$ \\
\hline & \multicolumn{3}{|c|}{ Being Exposed to a Terror Attack } & \multicolumn{3}{|c|}{ A Terror Attack in Israel } \\
\hline & Male $(n=105)$ & Female $(n=193)$ & $F$-Statistic & Male $(n=105)$ & Female $(n=193)$ & $F$-Statistic \\
\hline \multicolumn{7}{|l|}{ B. Israeli sample $(n=298)$} \\
\hline Emotional representations & 2.95 & 3.94 & $33.72^{* *}$ & 3.41 & 4.19 & $21.80^{* *}$ \\
\hline Likelihood & 2.54 & 3.15 & $18.98^{* *}$ & 5.81 & 5.80 & 0.02 \\
\hline Severity & 5.51 & 5.97 & $8.81^{*}$ & 3.90 & 5.11 & $45.24^{* *}$ \\
\hline
\end{tabular}

${ }^{*} p<0.01 ;{ }^{* *} p<0.001$

self-assessed knowledge $(\mathrm{M}=4.97, S D=1.63, F=$ $4.17, p<0.05)$. Israeli respondents with terror experiences perceived the terror risk more likely $(\mathrm{M}=$ $6.19, S D=1.08)$ when compared to those without terror experiences $(\mathrm{M}=5.65, S D=1.45, F=9.65$, $p<0.01$ ). Gender differences in mean ratings of TRPS scores for the Turkish and Israeli samples are presented in Table III. Findings were identical in both samples: (1) female respondents had higher emotional representations and severity perceptions compared to their male counterparts, and (2) females perceived being exposed to a terror attack to be more likely than males. No significant gender differences were found in perceived likelihood of a terror attack that may occur in the country. Based on these results, gender, experience with terror attacks, and perceived knowledge of terror attacks in the country were controlled in the main analyses.

\subsection{Risk Perceptions of Terror Attacks}

The differences in perceptions between personally being exposed to a terror attack and a terror attack that may occur in one's country were assessed by repeated measures multivariate analysis of covariance (MANCOVA). The demographic and background variables (i.e., gender, experience with terror attacks, and perceived knowledge of terror attacks in the country) were used as covariates. The results presented in Table IV indicate significant differences in the Turkish $(F=11.06, d f=3,530$, $p<0.001)$ and Israeli samples $(F=12.43, d f=$ $3,291, p<0.001)$. Both groups perceived the risk
Table IV. Mean Ratings and Standard Deviations (in

Parentheses) of Emotional Representations, Perceived Likelihood, and Severity of Being Personally Exposed to a Terror Attack Versus a Terror Attack in One's Country

\begin{tabular}{lcccc}
\hline & $\begin{array}{c}\text { Being } \\
\text { Exposed } \\
\text { to a Terror } \\
\text { Attack }\end{array}$ & $\begin{array}{c}\text { A Terror } \\
\text { Attack in } \\
\text { Turkey }\end{array}$ & $\begin{array}{c}F- \\
\text { Statistic }\end{array}$ & $\begin{array}{c}p \text { - } \\
\text { Value }\end{array}$ \\
\hline $\begin{array}{c}\text { A. Turkey }(n=536) \\
\quad \text { Emotional } \\
\quad \text { representations }\end{array}$ & $3.99(1.40)$ & $4.66(1.31)$ & 2.36 & 0.125 \\
$\quad \begin{array}{l}\text { Likelihood } \\
\text { Severity }\end{array}$ & $3.15(1.35)$ & $5.52(1.71)$ & 33.13 & 0.000 \\
$\begin{array}{l}\text { B. Israel }(n=298) \\
\text { Emotional } \\
\quad \text { representations }\end{array}$ & $6.10(1.17)$ & $6.04(1.31)$ & 0.45 & 0.502 \\
$\quad \begin{array}{l}\text { Likelihood } \\
\quad \text { Severity }\end{array}$ & $2.59(1.49)$ & $3.92(1.44)$ & 0.38 & 0.540 \\
\hline & $5.81(1.28)$ & $4.68(1.59)$ & 3.08 & 0.080 \\
\hline
\end{tabular}

of a terror attack that may occur in one's country more likely than the risk of being personally exposed to a terror attack. No significant personal-country differences were found in emotional representations and perceived severity ratings.

\subsection{Comparison of the Turkish and Israeli Samples on Risk Perceptions of Terror Attacks}

MANCOVA was applied to compare the TRPS scores in the Turkish and Israeli samples. Age and experience with terror attacks were used as covariates. The mean ratings of TRPS scores are reported in Table V. Turkish participants were found 
Table V. Means and Standard Deviations (in Parentheses) of TRPS Scores in the Turkish and Israeli Samples

\begin{tabular}{|c|c|c|c|c|}
\hline & $\begin{array}{l}\text { Turkey } \\
(n=536)\end{array}$ & $\begin{array}{c}\text { Israel } \\
(n=298)\end{array}$ & $\begin{array}{c}F- \\
\text { Statistic }\end{array}$ & $\begin{array}{c}p- \\
\text { Value }\end{array}$ \\
\hline \multicolumn{5}{|c|}{ A. Being personally exposed to a terror attack } \\
\hline $\begin{array}{l}\text { Emotional } \\
\text { representations }\end{array}$ & $3.99(1.40)$ & $3.59(1.49)$ & 5.33 & 0.021 \\
\hline Likelihood & $3.15(1.35)$ & 2.94(1.19) & 3.30 & 0.070 \\
\hline Severity & $6.10(1.17)$ & $5.81(1.28)$ & 9.84 & 0.002 \\
\hline \multicolumn{5}{|c|}{ B. A terror attack that may occur in one's country } \\
\hline $\begin{array}{l}\text { Emotional } \\
\text { representations }\end{array}$ & $4.66(1.31)$ & $3.92(1.44)$ & 33.49 & 0.000 \\
\hline Likelihood & $5.52(1.71)$ & $5.81(1.37)$ & 1.21 & 0.272 \\
\hline Severity & $6.04(1.31)$ & $4.68(1.59)$ & 98.00 & 0.000 \\
\hline
\end{tabular}

to be more emotional, and appeared to perceive the terrorism risk as more severe than the Israeli respondents. No significant difference was obtained between the two samples in perceived likelihood of terror attacks. These findings pertained both to personal and country risks.

\subsection{The Relationship Between Values and Risk Perceptions}

Pearson correlations between values and TRPS scores were computed in each sample. The mean of all value items was used as a covariate to control for individual differences in using the response scale. ${ }^{(26)}$ In addition, the demographic and background variables were also used as covariates. Tables VI and VII present the partial correlations between values and risk perceptions in the Turkish and Israeli samples, respectively.

The first research hypothesis predicted a negative relationship between self-direction values and emotional representations about being exposed to a terror attack. The findings in both samples supported the hypothesis. The more importance respondents attributed to self-direction values, the less emotional they felt about the risk for themselves.

The second research hypothesis predicted negative correlations between stimulation and self-direction values and perceived likelihood of being exposed to a terror attack. The findings partly supported this hypothesis. In both samples, "hedonism \& stimulation" values had significant negative correlations with perceived likelihood. The more priority participants gave to hedonism and stimulation values, the less they perceived the likelihood of being exposed to a terror attack.
However, correlations between self-direction values and likelihood judgments were not significant.

The second hypothesis also predicted positive correlations between tradition, conformity, and security values and perceived likelihood. Significant correlations were found between tradition and security values and perceived likelihood only in the Israeli sample but not in the Turkish sample. The more priority the Israeli respondents gave to tradition and security values, the more likely they perceived the risk for themselves. Benevolence and conformity values were not related to perceived likelihood in either sample.

The third research hypothesis predicted negative correlations between stimulation values and perceived likelihood of a terror attack that may occur in the country. This hypothesis was supported in both samples: the more importance respondents attributed to "hedonism \& stimulation" values, the less they perceived the likelihood of a terror attack that may occur in their country. The third hypothesis also predicted positive correlations between tradition, conformity, and security values and perceived likelihood of terror attacks. This was supported partly in the Israeli sample: the more importance the Israeli participants attributed to "benevolence \& conformity" and security values, the more likely they perceived the risk for their country. No significant correlations were obtained in either of the samples between self-direction and tradition values and perceived likelihood of the risk for the country.

The fourth research hypothesis predicted positive correlations between power and security values and perceived severity of being exposed to a terror attack. Conversely, negative correlations were predicted for universalism and benevolence values. The findings partly supported this hypothesis. Security values in the Turkish sample and universalism values in the Israeli sample correlated significantly with perceived severity about being personally exposed to a terror attack. The greater importance Turkish respondents attributed to security values, the more severe they perceived the risk for themselves. Correspondingly, the more priority the Israeli participants gave to universalism values, the less severe they perceived this risk. No significant correlations were found between power and "benevolence \& conformity" values and perceived severity in either sample.

The last research hypothesis predicted positive correlations between universalism, benevolence, and security values and perceived severity of a 
Table VI. Partial Correlations Between Values and Risk Perceptions in the Turkish Sample $(n=536)$

\begin{tabular}{|c|c|c|c|c|c|c|}
\hline & \multicolumn{3}{|c|}{ Being Personally Exposed to a Terror Attack } & \multicolumn{3}{|c|}{ A Terror Attack That May Occur in Turkey } \\
\hline & $\begin{array}{c}\text { Emotional } \\
\text { Representations }\end{array}$ & Likelihood & Severity & $\begin{array}{c}\text { Emotional } \\
\text { Representations }\end{array}$ & Likelihood & Severity \\
\hline Power & -0.02 & -0.04 & $\underline{0.01}$ & -0.01 & -0.05 & $\underline{0.00}$ \\
\hline Achievement & 0.00 & -0.01 & $\overline{0.02}$ & 0.02 & 0.06 & $\overline{0.02}$ \\
\hline Stimulation \& Hedonism & -0.08 & $\underline{-0.09 *}$ & -0.01 & $-0.11^{* *}$ & $\underline{-0.10^{*}}$ & -0.04 \\
\hline Self-Direction & $-0.11^{* *}$ & $\overline{0.03}$ & -0.04 & $-0.12^{* *}$ & $\overline{0.08}$ & $-0.13^{* *}$ \\
\hline Universalism & $\overline{-0.00}$ & $\overline{0.08}$ & $\underline{0.02}$ & 0.04 & 0.04 & $\underline{-0.03}$ \\
\hline Benevolence \& Conformity & -0.01 & $\underline{0.02}$ & $-\overline{0.01}$ & 0.05 & -0.03 & $\overline{0.10} *$ \\
\hline Tradition & $0.09^{*}$ & $\overline{0.02}$ & $\overline{-0.02}$ & -0.06 & $\overline{-0.05}$ & $\overline{0.02}$ \\
\hline Security & $0.09^{*}$ & $\overline{0.02}$ & $0.11^{* *}$ & $0.11^{* *}$ & $\overline{-0.03}$ & $\underline{0.15^{* *}}$ \\
\hline
\end{tabular}

Notes: Gender and perceived knowledge of terror attacks in one's country were controlled. Hypothesized correlations are underlined.

${ }^{* *} p<0.01 ;{ }^{*} p<0.05$; one-tailed for predicted associations, two-tailed for nonpredicted associations.

Table VII. Partial Correlations Between Values and Risk Perceptions in the Israeli Sample $(n=298)$

\begin{tabular}{|c|c|c|c|c|c|c|}
\hline & \multicolumn{3}{|c|}{ Being Personally Exposed to a Terror Attack } & \multicolumn{3}{|c|}{ A Terror Attack That May Occur in Israel } \\
\hline & $\begin{array}{c}\text { Emotional } \\
\text { Representations }\end{array}$ & Likelihood & Severity & $\begin{array}{c}\text { Emotional } \\
\text { Representations }\end{array}$ & Likelihood & Severity \\
\hline Power \& Achievement & 0.06 & -0.04 & $\underline{0.08}$ & 0.02 & $-0.18^{*}$ & $\underline{0.03}$ \\
\hline Stimulation \& Hedonism & -0.11 & $-0.15^{* *}$ & $\overline{0.01}$ & -0.09 & $-0.20^{* *}$ & $-\overline{0.02}$ \\
\hline Self-Direction & $-0.14^{* *}$ & $\overline{-0.01}$ & 0.07 & $-0.12^{*}$ & $\overline{0.04}$ & $-0.12^{*}$ \\
\hline Universalism & $\overline{-0.06}$ & $\overline{-0.09}$ & $-0.16^{* *}$ & -0.02 & 0.09 & -0.00 \\
\hline Benevolence \& Conformity & -0.04 & $\underline{0.09}$ & $\overline{-0.05}$ & -0.01 & $0.14^{* *}$ & $\overline{-0.00}$ \\
\hline Tradition & -0.09 & $0.24^{* *}$ & $-0.18^{*}$ & 0.09 & $-\overline{0.06}$ & 0.07 \\
\hline Security & $0.18^{*}$ & $\overline{0.17}^{* *}$ & $\underline{0.03}$ & $0.19^{* *}$ & $\overline{0.14}^{* *}$ & $\underline{0.07}$ \\
\hline
\end{tabular}

Notes: Partial correlations are controlling gender, experience with terror attacks, and perceived knowledge of terror attacks in one's country were controlled. Hypothesized correlations are underlined.

${ }^{* *} p<0.01 ;{ }^{*} p<0.05$; one-tailed for predicted associations, two-tailed for nonpredicted associations.

terror attack that may occur in the country. This hypothesis was partly supported for the Turkish sample but not supported for the Israeli sample. The greater importance Turkish respondents attributed to "benevolence \& conformity" and security values, the higher they perceived the severity of a terror attack that may occur in their country. The correlation between universalism values and perceived severity in the Turkish sample was not significant. Finally, the analyses yielded several unpredicted results regarding relationships between values and risk perceptions. The unpredicted significant (two-tailed) correlations that were found in both samples were as follows. (i) Security values were significantly correlated with emotional representations. The more importance respondents gave to security values, the more emotional they felt about terror risks. (ii) Self-direction values demonstrated significant correlations with emotional representations about a terror attack that may occur in the country. The greater priority participants attributed to self-direction values, the less emotional they felt about the risk for their country. (iii) Self-direction values exhibited significant correlations with perceived severity of a terror attack that may occur in the country. The more importance participants attributed to self-direction values, the lower was their perceived severity of a terror attack that may occur in their countries.

The directions of the predicted and unpredicted findings in both samples are presented in Table VIII. The findings suggest that self-direction values are negatively related to emotional representations of terror attacks, whereas security values are positively correlated with emotions; "hedonism \& stimulation" values are negatively related to perceived likelihoods of terror attacks; self-direction values were negatively related to severity perceptions about a terror attack that may occur in the country. 
Table VIII. Summary About the Directions of Significant Correlations Found Between Values and Risk Perceptions

\begin{tabular}{|c|c|c|c|c|c|c|}
\hline & \multicolumn{3}{|c|}{ Being Personally Exposed to a Terror Attack } & \multicolumn{3}{|c|}{ A Terror Attack That May Occur in One's Country } \\
\hline & $\begin{array}{c}\text { Emotional } \\
\text { Representations }\end{array}$ & Likelihood & Severity & $\begin{array}{c}\text { Emotional } \\
\text { Representations }\end{array}$ & Likelihood & Severity \\
\hline $\begin{array}{l}\text { Power \& } \\
\text { Achievement }\end{array}$ & & & & & Negative in IL & \\
\hline $\begin{array}{c}\text { Stimulation \& } \\
\text { Hedonism }\end{array}$ & Negative in IL & Negative & & Negative in $T R$ & Negative & \\
\hline Self-Direction & Negative & & & Negative & & Negative \\
\hline Universalism & & & Negative in IL & & & \\
\hline $\begin{array}{c}\text { Benevolence \& } \\
\text { Conformity }\end{array}$ & Positive in $T R$ & & & & Positive in IL & Positive in TR \\
\hline Tradition & Positive in $T R$ & Positive in IL & Negative in IL & & & \\
\hline Security & Positive & Positive in IL & & Positive & Positive in IL & Positive in $T R$ \\
\hline
\end{tabular}

Notes: Bold for identical findings in both countries; italic for one country. TR: Turkish sample, IL: Israeli sample.

\section{DISCUSSION}

\subsection{Risk Perceptions}

Significant associations were found between gender and terror risk perception in both samples. Females reported more negative emotions and had higher severity perceptions than their male counterparts about personally being exposed to a terror attack and a terror attack that may occur in their country. In addition, their perceived likelihood of being exposed to a terror attack was higher than that of the males. These results replicate gender differences in terror risk perceptions found in previous research. In particular, Turkish and Israeli females were reported as perceiving more costs (severe consequences) and vulnerability, as well as expressing more negative affect about the risk of terror attacks. ${ }^{(12)}$ Similarly, higher perceived risk of terror attacks was associated with the female gender in Britain, ${ }^{(27)}$ and gender differences in emotions were associated with gender differences in perceived terror risk in the United States. ${ }^{(28)}$

Turkish and Israeli participants perceived the likelihood of terror risk for their country as being significantly higher than its likelihood for themselves. However, no significant differences were found between these risks in emotional representations and perceived severity ratings. The difference in the perceived likelihood ratings may be explained by the availability heuristic, which refers to the tendency assessing the probability of an event by the ease with which relevant instances come to mind. ${ }^{(29)}$

Earlier studies conducted in Canada and Sweden also found higher perceived risk for the country compared to oneself. ${ }^{(30,31)}$ Therefore, our finding may also be explained by unrealistic optimism, which refers to the tendency of people to report that they are less likely than others to experience negative events. ${ }^{(32)}$ It can result from self-protective processes aimed to protect self-esteem, to enhance a positive social image, or to reduce anxiety. ${ }^{(32-34)}$ It can also result from unmotivated cognitive processes and judgmental biases ${ }^{(35)}$ such as the illusion of control. ${ }^{(36)}$ The literature on unrealistic optimism has mainly focused on vulnerability perceptions rather than severity, although researchers in this field define risk perception as comprising both vulnerability and severity. One exception is a study that provided findings compatible with ours, such that vulnerability ratings increased as levels of dissimilarity between oneself and a referent increased, but this pattern was not observed for severity ratings. ${ }^{(37)}$

A discussion about the similarity in the emotional representations and perceived severity ratings is also interesting in its own right but it is in the scope of another publication.

\subsection{Cross-Cultural Comparisons}

Our finding that shows a significant difference between the two samples in security values (i.e., Turkish participants attributed greater priority to security values compared to their Israeli counterparts) contradicts with the findings of past studies conducted by Schwartz and Bardi. ${ }^{(15,38)}$ These studies did not report any differences between the Turkish and Israeli student samples. We speculate that the contradiction may result from the size and composition of the samples. In the past studies, the sample sizes were smaller than those in the current research. 
In addition, Israeli samples consisted of students living in the area of Jerusalem, which can be considered as less secular/liberal than Tel Aviv. Another speculation can be a methodological difference between the current and past research. We administered the SVS before the TRPS to half of the samples, and the TRPS before the SVS to the other half, whereas SVS was always administered before other surveys in past studies. Because we controlled the effect of order in the analysis, we do not expect any framing effect on our results. The different findings provide a promising venue for further comparative studies.

Comparison of the Turkish and Israeli samples on risk perception showed similarities and differences between the two cultures. The Turkish participants were more emotional than the Israeli respondents about being exposed to a terror attack and a terror attack that may occur in one's country. This result replicates the findings of a previous study. ${ }^{(12)}$ The differences in emotions between the two samples may result from the sociocultural factors that predict the subjective experience of emotions. ${ }^{(39)}$ However, this argument needs further support from other studies that show the emotional difference between the two countries more generally, not just regarding terror attacks. At least to our knowledge, there are no studies highlighting this difference between Turkey and Israel.

No significant difference was found between the two samples in perceived likelihood of these risks. This result supports the view that risks have a common universal dimensional structure, although the cultures may differ with regard to the salience of associated factors. ${ }^{(12,40)}$ Overall, the present findings about terror risk perception support the views presented by the SARF about social processes that affect representations of risks by individuals and groups across different cultural/societal contexts.

\subsection{Terror Risk Perceptions and Values}

Five hypotheses were postulated to investigate the relationships between values and risk perception of terror attacks. We found weak but significant correlations between values and risk perception. The findings are summarized in three groups: (1) predicted associations that revealed a consistent pattern in both samples, (2) unpredicted associations that revealed a consistent pattern in both samples, and (3) predicted associations that were supported in one of the samples.
As hypothesized, self-direction values correlated negatively with emotional representations about being personally exposed to a terror attack. The finding that a negative correlation was also obtained for a terror attack in the country was not predicted, but may be explained by the concept of self-control. Since self-direction values derive from organismic needs for control and mastery, ${ }^{(14)}$ it may be argued that they are part of the higher-order personality trait dimension "self-control," obtained in factor analysis of the big five personality traits. ${ }^{(41,42)}$ Self-control reflects interpersonal self-control, taskoriented self-control, and emotional self-control that helps individuals to be adaptive to external threat, imminent danger, and potential loss. Extant literature indicates that self-control traits are related to less negative affect. ${ }^{(43)}$ Accordingly, self-control through the measure of self-direction values may have helped participants in both our samples to control distressing emotions about a terror risk, both at personal and country levels.

As hypothesized, significant negative correlations were found between "hedonism \& stimulation" values and perceived likelihood of terror attacks. However, self-direction values did not exhibit significant correlations with perceived likelihood in either sample. Hedonism and stimulation values together with self-direction values express people's readiness to accept and desire to pursue new, challenging, and uncertain outcomes. ${ }^{(17)}$ Although these values together emphasize openness to change, the organismic needs from which they are derived are different. They originate from organismic needs for variety, stimulation, and pleasure; however, self-direction values stem from organismic needs for control and mastery. ${ }^{(14)}$ It may be argued that the emphasis of hedonism and stimulation values on affective pleasure decreases perceived likelihood of a terror attack, whereas the focus of self-direction values on control and mastery decreases emotional representations.

Our findings regarding "hedonism \& stimulation" values are congruent with the sensation seeking literature. Sensation seeking is "a trait defined by the seeking of varied, novel, complex, and intense sensations and experiences, and the willingness to take physical, social, legal, and financial risks for the sake of such experience." ${ }^{(44)}$ High-sensation seekers were found to appraise the environment as less threatening than low-sensation seekers. ${ }^{(4)}$ Specifically, in one study high-sensation seekers rated their risk of being a victim of a crime or a violent act as lower compared to low-sensation seekers. ${ }^{(46)}$ Given 
the similarities between "hedonism \& stimulation" values and sensation seeking, the current results may be viewed as supporting previous findings.

An unpredicted finding showed that security values correlated positively with emotional representations regarding the risk of terror attacks. Security values emphasize safety, harmony, and stability of society/relationships and oneself. Across seven samples, security values correlated positively and consistently with safety/health microworries (e.g., my getting cancer) and macroworries (e.g., the outbreak of a nuclear war). ${ }^{(17)}$ Therefore, high importance given to security values may also sensitize people's feelings about the risk of terror attacks. It should be noted that these unpredicted findings deserve further replications.

Differences between the samples in the associations of values with risk perceptions may have theoretically interesting implications. For example, security and benevolence and conformity values were associated with perceived severity in the Turkish sample, but correlated with perceived likelihood in the Israeli sample. Cultural differences in values and in normative expressions of risk perception may underlie these differences and deserve future research.

\subsection{Limitations of the Study}

Participants in this study were university students from Turkey and Israel. It is possible that their value structures and perceptions of terror risk do not represent those of people from different demographic and socioeconomic backgrounds in their countries. It is, therefore, important to be cautious in making overgeneralizations from these findings. Further research using the current instruments in other groups with differing demographics is highly desirable.

The current research focused on two cognitive components of risk perception: perceived likelihood and perceived severity. Future studies will benefit from investigating the relationship between values and other cognitive variables associated with perceived risk of terror, like trust and control. ${ }^{(12)}$ Such research will increase our understanding of the complicated social-cognitive mechanisms behind risk perception.

\section{CONCLUSIONS AND IMPLICATIONS}

This study involved: (1) incorporating $\operatorname{SVT}^{(13,14)}$ into the risk perception research, and (2) examining the relationship between basic human values and risk perception of terror attacks in two countries, Turkey and Israel. The consistency of the associations between risk perception and the self-direction, "hedonism \& stimulation," and security values across the two samples provides support for the existence of a link between basic values and risk perception of terror attacks. This consistent pattern of associations suggests the applicability of $\operatorname{SVT}^{(13,14)}$ in explaining risk perceptions. The TRPS in its current form could be expanded to include more cognitive components of risk perception (e.g., trust and control). Accordingly, the associations of values with risk perceptions need to be further investigated in other cultures to assess the cross-cultural generalization of the present findings.

Our finding that people who endorse certain values may be perceiving terror risks differently than others has potentially important ramifications for tailoring relevant communication messages for different target audiences. For example, people attributing more importance to security values may require informative messages focused on reducing their perceived risk likelihoods, whereas those giving more importance to "hedonism \& stimulation" and self-direction values may require more alerting messages to better prepare them for a potential terror attack. It is important to identify differences in risk perceptions and values within and between groups when creating communication messages. Getting support from several individuals or groups is necessary to achieve a consensus regarding a course of action. Not all of them would endorse but valuing their perceptions and values in communications can help to maintain trust and credibility and thereby increase community support for the proposed course of action. Perceptions of risk by public officials also appear to play an important role in the examination of a nation's preparedness to deal with terror risk. ${ }^{(47)}$ In designing and implementing training programs for professionals to provide effective emergency risk communication to an anxious public, it is important to consider their values. Future research on the relationship between security professionals' values and their risk perceptions of terror attacks may shed more light on this critical issue.

In an earlier study aimed to demonstrate the effects of emotions on policy endorsement, it was reported that, after the 9/11 attacks, people whose emotional reactions were dominated by anger attributed the attacks to fanaticism of the terrorists and to poor U.S. security, thus endorsing an 
aggressive military response, whereas those whose emotional reactions were dominated by sadness or fear expressed reservations about a strong military reaction. ${ }^{(48)}$ A similar relationship can be predicted between values and policy preferences. Thus, people attributing more importance to security values may assert more punitive preferences, whereas those giving more priority to "hedonism \& stimulation" and self-direction values may assert more conciliatory preferences. The current study has shown that values may be instrumental in gauging risk perceptions regarding terrorism. Further research examining the effects of value preferences on policy endorsement concerning terrorism may support policymakers' efforts to communicate effective and targeted messages to a risk-conscious public.

\section{ACKNOWLEDGMENTS}

This research was supported in part by a grant (Suleyman Demirel program for contemporary Turkish studies) from the Moshe Dayan Center, Tel Aviv University and Academic Research Program of the Israeli Foreign Ministry, and by a research award (Prof. Dr. Orhan Karacadağ Award for Scientific Achievement) from the Faculty of Business Administration, Bilkent University to Gülbanu Kaptan. We are grateful to Shalom Schwartz and Sonia Roccas for their invaluable guidance. We wish to express our sincere thanks to Baruch Fischhoff, Yael Benyamini, Deniz Sahin, Zahide Karakitapoglu-Aygun, and two anonymous referees for their constructive comments.

\section{REFERENCES}

1. Huddy L, Feldman S, Capleos T, Provost C. The consequences of terrorism: Distangling the effects of personal and national threat. Political Psychology, 2002; 23:485-507.

2. Kasperson RE, Renn O, Slovic P, Brown HS, Emel J, Goble $\mathrm{R}$, Kasperson JX, Ratick S. The social amplification of risk. Risk Analysis, 1988; 8:177-187.

3. Gigerenzer G. Dread risk, September 11, and fatal traffic accidents. Psychological Science, 2004; 15:286-287.

4. Fischhoff B. Behaviorally realistic risk management. Pp. 7788 in Daniels RJ, Kettl DF, Kunreuther H (eds). On Risk and Disaster: Lessons from Hurricane Katrina. Philadelphia: University of Pennsylvania Press, 2006.

5. Pidgeon N, Hood C, Jones D, Turner B, Gibson R. Risk perception. Pp. 89-134 in Royal Society Study Group, Risk: Analysis, Perception and Management. London: Royal Society, 1992.

6. Douglas M, Wildawsky A. Risk and Culture. Berkeley and Los Angeles, CA: University of California Press, 1982.

7. Dake K. Orienting dispositions in the perception of risk: An analysis of contemporary worldviews and cultural biases. Journal of Cross-Cultural Psychology, 1991; 22(1):61-82.
8. Dake K. Myths of nature: Culture and the social construction of risk. Journal of Social Issues, 1992; 48:31-37.

9. Peters E, Slovic P. The role of affect and world-views as orienting dispositions in the perception and acceptance of nuclear power. Journal of Applied Social Psychology, 1996; 26:1427-1453.

10. Palmer CGS. Risk perception: An empirical study of the relationship between worldview and the risk construct. Risk Analysis, 1996; 16:717-723.

11. Marris, C, Langford IH, O'Riordan T. A quantitative test of the cultural theory of risk perceptions: Comparison with the psychometric paradigm. Risk Analysis, 1998; 18:635-647.

12. Shiloh, S, Güvenç G, Önkal D. Cognitive and emotional representations of terror attacks: A cross-cultural exploration. Risk Analysis, 2007; 27(2):397-409.

13. Schwartz SH. Universals in the content and structure of values: Theoretical advances and empirical tests in 20 countries. Pp. 251-265 in Zanna M (ed). Advances in Experimental Social Psychology 25. Orlando, FL: Academic, 1992.

14. Schwartz SH. Are there universal aspects in the structure and contents of human values. Journal of Social Issues, 1994; 50(4):19-46.

15. Schwartz SH. Basic human values: Their content and structure across countries. Pp. 21-55 in Tamayo A, Porto JB (eds). Valores e comportamento nas organizações [Values and Behavior in Organizations]. Petrópolis, Brazil: Vozes, 2005.

16. Roccas S, Sagiv S, Schwartz SH, Knafo A. The big five personality factors and personal values. Personality and Social Psychology Bulletin, 2002; 28(6):789-801.

17. Schwartz SH, Sagiv L, Boehnke K. Worries and values. Journal of Personality, 2000; 68(2):309-346.

18. Pyszczynski T, Solomon S, Greenberg J. In the Wake of 9/11: The Psychology of Terror. Washington, DC: APA Publications, 2003.

19. Holtgrave D., Weber EU. Dimensions of risk perception for financial and health risks. Risk Analysis, 1993; 13:553-558.

20. Schwartz SH. Basic values: How they motivate and inhibit prosocial behavior. In Mikulincer M, Shaver P (eds). Herzliya Symposium on Personality and Social Psychology, Vol. 1. Washington: American Psychological Association Press, 2009.

21. Mikulincer M, Gillath O, Sapir-Lavid Y, Yaakobi E, Arias K, Tal-Aloni L, Bor G. Attachment theory and concern for others' welfare: Evidence that activation of the sense of secure base promotes endorsement of self-transcendence values. Basic and Applied Social Psychology, 2003; 25(4):299-312.

22. Sagiv L, Schwartz SH. Value priorities and subjective wellbeing: Direct relations and congruity effects. European Journal of Social Psychology, 2000; 30:177-198.

23. Bilsky W, Schwartz SH. Values and personality. European Journal of Personality, 2006; 8(3):163-181.

24. Borg I, Shye S. Facet Theory: Form and Content. London: Sage, 1995

25. Guttman L. A general nonmetric technique for finding the smallest coordinate space for a configuration of points. Psychometrica, 1968; 33:469-506.

26. Schwartz SH. Robustness and fruitfulness of a theory of universals in individual human values. Pp. 56-95 in Tamayo A, Porto JB (eds). Valores e comportamento nas organizações [Values and Behavior in Organizations]. Petrópolis, Brazil: Vozes, 2005

27. Goodwin R, Willson M, Gaines S. Terror threat perception and its consequences in contemporary Britain. British Journal of Psychology, 2005; 96:389-406.

28. Lerner JS, Gonzalez RM, Small DA, Fischhoff B. Effects of fear and anger on perceived risks of terrorism: A national field experiment. Psychological Science, 2003; 14:144-150.

29. Tversky A, Kahneman D. Judgment under uncertainty: Heuristics and biases. Science, 1974; 185(4157):1124-1131. 
30. Lemyre L, Turner MC, Schwartz SH, Lee JEC, Krewski D. Public perception of terrorism threats and related information sources in Canada: Implications for the management of terrorism risks. Journal of Risk Research, 2006; 9(7): $755-774$.

31. SSE/EFI Working Paper Series in Business Administration No 2002:11. The Perceived Risk of Terrorism. Available at: http://swoba.hhs.se/hastba/papers/hastba2002 011.pdf, Accessed on February 28, 2012.

32. Weinstein ND. Unrealistic optimism about future life events. Journal of Personality and Social Psychology, 1980; 39:806820.

33. Weinstein ND. Why it won't happen to me: Perceptions of risk factors and susceptibility. Health Psychology, 1984; 3:431-457.

34. Joffe H. Risk and the Other. Cambridge, UK: Cambridge University Press, 1999.

35. Weinstein ND. Unrealistic optimism about susceptibility to health problems. Journal of Behavioral Medicine, 1982; 5:441-460.

36. Harris P. Sufficient grounds for optimism? The relationship between perceived controllability and optimistic bias. Journal of Social and Clinical Psychology, 1996; 15:9-52.

37. Rimal RN, Morrison D. A uniqueness to personal threat (UPT) hypothesis: How similarity affects perceptions of susceptibility and severity in risk assessment. Health Communication, 2006; 20(3):209-219.

38. Schwartz SH, Bardi A. Value hierarchies across cultures. Journal of Cross Cultural Psychology, 2001; 32(3):268-290.

39. Basabe N, Paez D, Valencia J, Rime B, Pennebaker J, Diener E, Gonzalez JL. Sociocultural factors predicting subjective experience of emotion: A collective level analysis. Psicothema, 2000; 12:S389-S406.

40. Slovic P. Perception of risk: Reflections on the psychometric paradigm. Pp. 117-152 in Krimsky S, Golding D (eds). Social Theories of Risk. New York: Praeger, 1992.

41. Digman JM. Higher-order factors of the big five. Journal of Personality and Social Psychology, 1997; 23:1246-1256.

42. Carroll J. The five-factor personality model: How complete and satisfactory is it. Pp. $97-126$ in Braun H, Jackson D, Wiley D (eds). The Role of Constructs in Psychological and Educational Measurement. Mahwah, NJ: Lawrence Erlbaum, 2002.

43. Olson KR. Engagement and self-control: Superordinate dimensions of big five traits. Personality and Individual Differences, 2005; 38:1689-1700.

44. Zuckerman M. Behavioral Expressions and Biosocial Bases of Sensation Seeking. New York: Cambridge Press, 1994.

45. Roberti P. A review of behavioral and biological correlates of sensation-seeking. Journal of Research in Personality, 2004; 38:256-279.

46. Horvath P, Zuckerman M. Sensation seeking, risk appraisal and risky behavior. Personality and Individual Differences, 1993; 14:41-52.

47. Slovic P, Weber E. Perception of Risk Posed by Extreme Events. Paper presented at the Conference on Risk Management Strategies in an Uncertain World, held in Palisades, New York, April 12-13, 2002.

48. Sadler MS, Lineberger M, Correll J, Park B. Emotions, attributions, and policy endorsement in response to the September $11^{\text {th }}$ terrorist attacks. Basic and Applied Social Psychology, 2005; 27:249-258. 